\title{
Graph representation of $\boldsymbol{n}$-dimensional space
}

\author{
Tomasz Kosicki
}

Received: 14 January 2014/ Accepted: 14 February 2014/Published online: 13 March 2014

(C) Shanghai University and Springer-Verlag Berlin Heidelberg 2014

\begin{abstract}
This paper investigates how graph representation can be created for the mesh which is a discrete approximation of $n$-dimensional continuous space. The paper discusses the relationship between mesh dimensionality and the type and quantity of edges connecting each vertex with its neighbors. Basing on the analysis, a simple algorithm is also proposed to create such graph representation. The purpose of the graph is to search optimal paths and trajectories in the represented space.
\end{abstract}

Keywords Trajectory optimization - Path optimization . Graph search algorithms

\section{Introduction}

Path and trajectory optimization (trajectory is a dynamically constrained path) are desired for constantly growing number of applications. They are among the most basic tasks in the variety of robotic fields, such as mobile, industrial, space, underwater, aerial, and tele-robotics, as well as beneficial in many other fields, such as movie and game industry, and virtual prototyping [1]. Potentially they are useful in any system which can be modeled by control theory, ranging from electric engineering to computational economics.

Path and trajectory optimization are usually performed by means of a graph, which is either an exact or approximate representation of a real system. The exact representation can be created for discrete systems, such as the

T. Kosicki $(\bowtie)$

Department of Production and Quality Engineering, Norwegian

University of Science and Technology, Trondheim, Norway

e-mail: tomasz.kosicki@ntnu.no system of cities connected by roads which is used in logistics [2], whereas the approximate representation is created for continuous systems, such as configuration space of an industrial robot [3]. The purpose of the optimization is to find optimal, suboptimal, or at least feasible path, between initial and goal vertices in the graph, which corresponds to initial and goal states in the system. While for discrete systems it is always possible to find optimal path, when it exists in the represented system (completeness), for continuous system it depends on the sampling method and resolution used to create its graph representation (probabilistic or resolution completeness) [4].

For continuous systems, one can distinguish three subtasks of the optimization: sampling space, creating representation, and searching optimal solution. The subtasks can be accomplished, either one after another, or iteratively in a feedback loop. There is a considerable number of methods available to be applied for the subtasks, which are well summarized in Ref. [5]. This article is concentrated on the second subtask. It analyses how a mesh, which was created by uniform deterministic sampling of $n$-dimensional space [6], can be represented by a graph, and proposes simple algorithm to create the graph. Though there are more efficient sampling methods, the used one is among the simplest to implement. Moreover, it is also convenient to analyze the relation between the mesh and graph, which can be used further for more sophisticated algorithms and sampling methods.

The paper is structured as follows. First, mathematical concepts relevant for further investigation are briefly explained in the preliminaries. Then, the graph representation of $n$-dimensional mesh is analyzed. Subsequently, an algorithm to create the representation is proposed and demonstrated for a simple binary 2-dimensional mesh $3 \times 3$. Finally, a conclusion is given. 


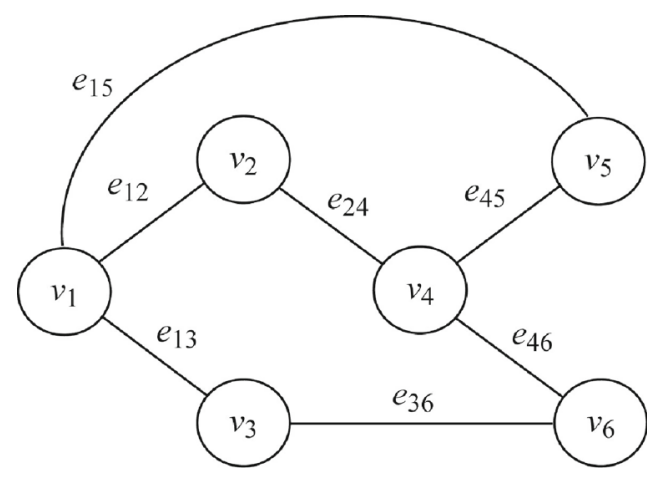

Fig. 1 Undirected graph and its adjacency matrix

\section{Mathematical preliminaries}

\subsection{Graph}

A graph $G=(V, E)$ consists of finite nonempty sets of vertices $V$ and edges $E$. If each edge is a set $\{v, w\}$ of vertices, the graph is undirected. Whereas if each edge is an ordered pair $[v w]$ of vertices, the graph is directed [7].

\subsubsection{Adjacency and weight matrices}

A graph can be represented by means of adjacency matrix $\boldsymbol{A}=|V| \times|V|$. The matrix has elements $\boldsymbol{A}[i, j]=1$ if there is an edge from vertex $i$ to vertex $j$, and $\boldsymbol{A}[i, j]=0$ if there is no edge [7]. Adjacency matrix can be also considered as a special case of distance matrix $\boldsymbol{D}=|V| \times|V|$ which has the same unit distance for all edges.

A distance (or weight) matrix has elements $\boldsymbol{D}[i, j]=d_{i j}$ if there is an edge from vertex $i$ to vertex $j$, and the distance between the vertices (or the weight of the edge) is a metric $d_{i j}=\left\|\left(v_{i}, v_{j}\right)\right\|$. Similarly as for adjacency matrix, $\boldsymbol{D}[i, j]=$ 0 if there is no edge [7].

For an undirected graph both adjacency and distance matrices are symmetric. Since usually the elements of matrices are zeroes (see Fig. 1), it is convenient in computer science to store only non-zero elements, and in particular for undirected graph, to store only the elements below main diagonal. The data structure used for this purpose is called a sparse matrix, which is $\operatorname{sparse}(\boldsymbol{A})=$ $[\{(i, j): A[i, j] \neq 0\}, \cdots][8]$.

\subsubsection{Path, length of path, and weight of path}

A path in a graph is a sequence of edges $\left(v_{1}, v_{2}\right),\left(v_{2}\right.$, $\left.v_{3}\right), \cdots,\left(v_{n-1}, v_{n}\right)$.

The length of a path is equal to the number of edges in the path $l_{1, n}=n-1$.

The weight of a path is equal to $w_{1, n}=$ $\sum_{i=1}^{n-1}\left\|\left(v_{i}, v_{i+1}\right)\right\|$.
If adjacency and weight matrices are identical, the weight of a path is equal to the length of the path.

$2.2 n$-cube, $n$-rectangle, and $n$-mesh

An $n$-cube (or hypercube) is an $n$-dimensional analogue of a square (2-cube) and a cube (3-cube). For a unit $n$-cube, diagonals in $r$-dimensions $r=1,2, \cdots, n$ are equal to $\sqrt{k}$ [9] and abbreviated as $r$-agonals.

Each $n$-cube with $n>0$ is composed of $E_{n-1, n}$ elements which are (n-1)-cubes. The number of elements (see Table 1) is determined by the formula [10]:

$E_{m, n}=\left\{\begin{array}{cl}1, & n=0, \\ 2^{n-m}\left(\begin{array}{c}n \\ m\end{array}\right), & n>0,\end{array} \quad m=0,1, \cdots, n\right.$.

An $n$-rectangle (or hyperrectangle) is an $n$-dimensional analogue of a rectangle (2-rectangle) and a cuboid (3rectangle), whereas $n$-dimensional mesh (also $n$-mesh or hypermesh) is a discrete representation of $n$-rectangle.

\subsection{Manifolds}

In simple words, $n$-dimensional topological manifold (abbreviated as $n$-manifold) can be explained as a subset of Euclidean space $\mathbf{R}^{k}$ that is locally Euclidean of dimensions $n$ [11] for $n \leq k$. For example, the surface of Earth is a sphere which is 2-manifold. While the points on Earth's surface are a subset of $\mathbf{R}^{3}$, locally they can be considered as Euclidean $\mathbf{R}^{2}$, since Earth's curvature usually cannot be perceived from its surface. Other manifolds which are subsets of $\mathbf{R}^{3}$ can be also easily visualized, e.g., 1-manifolds as curves, 2-manifolds as surfaces (see Fig. 2), and the only one 3-manifold, Euclidean space $\mathbf{R}^{3}$, as Euclidean space $\mathbf{R}^{3}$.

From engineering point of view, $n$-manifold is an object with $n$ degrees of freedom (DOF) such as $n$-dimensional state space. For example, configuration space of 2DOF planar manipulator with unconstrained joint positions is a 2-torus 
Table $1 E_{m, n}$ elements of $n$-cube, where $n=1,2, \cdots, 6$

\begin{tabular}{|c|c|c|c|c|c|c|c|}
\hline \multirow[t]{2}{*}{$n$} & \multicolumn{7}{|l|}{$m$} \\
\hline & $\begin{array}{l}0 \\
\text { Vertices }\end{array}$ & $\begin{array}{l}1 \\
\text { Edges }\end{array}$ & $\begin{array}{l}2 \\
\text { Faces }\end{array}$ & $\begin{array}{l}3 \\
\text { Cells }\end{array}$ & $\begin{array}{l}4 \\
4 \text {-faces }\end{array}$ & $\begin{array}{l}5 \\
5 \text {-faces }\end{array}$ & $\begin{array}{l}6 \\
6 \text {-faces }\end{array}$ \\
\hline 0 Point & 1 & & & & & & \\
\hline 1 Line segment & 2 & 1 & & & & & \\
\hline 2 Square & 4 & 4 & 1 & & & & \\
\hline 3 Cube & 8 & 12 & 6 & 1 & & & \\
\hline 4 Tesseract & 16 & 32 & 24 & 8 & 1 & & \\
\hline 5 Penteract & 32 & 80 & 80 & 40 & 10 & 1 & \\
\hline 6 Hexeract & 64 & 192 & 240 & 160 & 60 & 12 & 1 \\
\hline
\end{tabular}

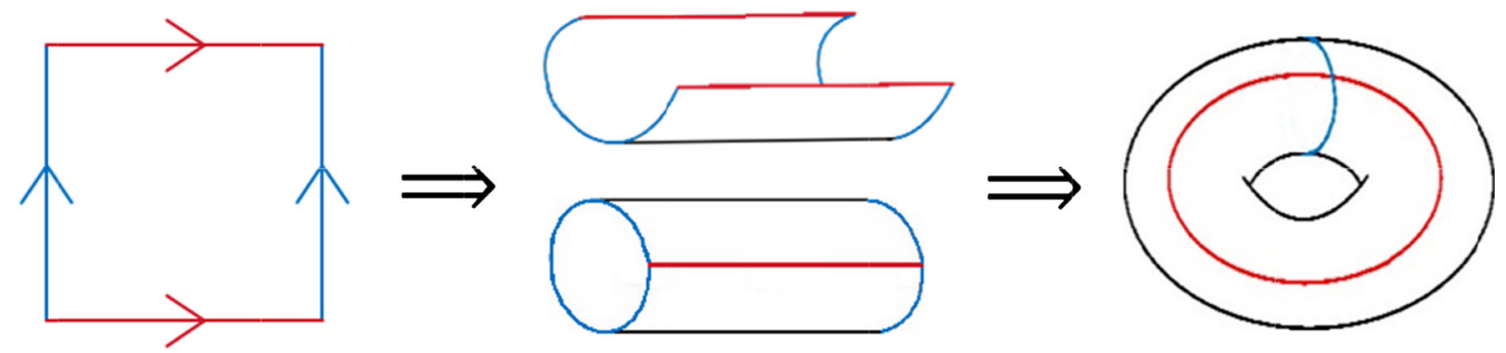

Fig. 2 Euclidean space $\mathbf{R}^{2}$ folded along 2 axes can be visualized as 2-torus

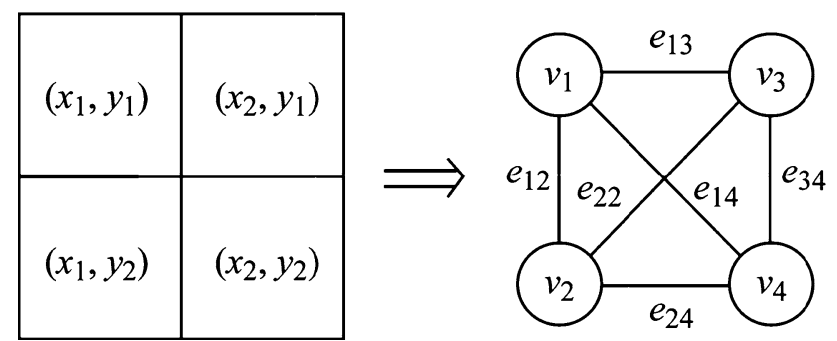

Fig. 32 -mesh $2 \times 2$ can be represented by an undirected graph $G_{2 \times 2}=(V, E)$

(see Fig. 2), and of 7DOF redundant industrial robot is a 7-torus. Further in the article, such manifolds, when discretized into $n$-mesh, are referred as folded $n$-mesh.

\section{Graph representation of $n$-mesh}

\subsection{Simple 2-mesh $2 \times 2$}

A 2-mesh $2 \times 2$ consists of 4 samples, which can be visualized as squares (see Fig. 3). If one considers the samples to be vertices connected with neighborhooding samples by edges, one obtains an undirected graph with 4 vertices and 6 edges (see Fig. 3):
$\left\{\begin{array}{l}G_{2 \times 2}=(V, E) \\ V=\left\{v_{1}, v_{2}, v_{3}, v_{4}\right\} \\ E=\left\{e_{12}, e_{13}, e_{14}, e_{23}, e_{24}, e_{34}\right\}\end{array}\right.$

One can distinguish between "orthogonal" and "diagonal" edges, which can be further divided into edges parallel to versors $\hat{x}, \hat{y}$, and parallel to the resultants $\hat{x}+\hat{y}$ and $\hat{x}-\hat{y}$ :

$$
\left\{\begin{array}{l}
E=E_{C 1}^{2} \cup E_{C 2}^{2}=\left\{e_{12}, e_{13}, e_{24}, e_{34}\right\} \cup\left\{e_{14}, e_{23}\right\}, \\
E_{C 1}^{2}=E_{\hat{x}} \cup E_{\hat{y}}=\left\{e_{13}, e_{24}\right\} \cup\left\{e_{12}, e_{34}\right\}, \\
E_{C 2}^{2}=E_{\hat{x}+\hat{y}} \cup E_{\hat{x}-\hat{y}}=\left\{e_{14}\right\} \cup\left\{e_{23}\right\} .
\end{array}\right.
$$

The graph representing 2-mesh $2 \times 2$ has therefore 2 distinctive edge classes, which can be further divided into 2 subclasses, and the total number of edge subclasses is 4 :

$$
\left\{\begin{array}{l}
\overline{\overline{\overline{E_{C}^{2}}}}=\overline{\overline{\left\{E_{C 1}^{2}, E_{C 2}^{2}\right\}}}=2, \\
\overline{\overline{E_{C 1}^{2}}}=\overline{\overline{\left\{E_{\hat{x}}, E_{\hat{y}}\right.}}=\overline{\overline{E_{C 2}^{2}}}=\overline{\overline{\left\{E_{\hat{x}+\hat{y}}, E_{\hat{x}-\hat{y}}\right\}}}=2, \\
\overline{\overline{U E_{C}^{2}}}=\sum_{k=1}^{n} \overline{\overline{E_{k}^{n}}}=4, n=2
\end{array}\right.
$$

Further, there is used short notation $E_{C}^{n}=$ $\left\{\overline{\overline{E_{C 1}^{n}}}, \overline{E_{C 2}^{n}}, \cdots, \overline{\overline{E_{C n}^{n}}}\right\}$, where $n$ is the number of edge classes. For example, the graph representation of 2-mesh has $E_{C}^{2}=\{2,2\}$. 


\subsection{Arbitrary $n$-mesh}

Each $n$-mesh can be decomposed to a set of $n$-cubes representing samples of discretized space. In particular, 2- and 3-meshes can be decomposed to easily imaginable squares and cubes. One can, therefore, obtain the sets $E_{C}^{2}$ and $E_{C}^{3}$ simply by counting uniquely oriented 1- and 2-agonals for square $E_{C}^{2}=\{2,2\}$, and 1-, 2-, and 3-agonals for cube $E_{C}^{3}=\{3,6,4\}$.

\subsubsection{Edge classes $E_{C}^{n}$}

Since $\overline{\overline{E_{C}^{1}}}=1$ for $n=1, \overline{\overline{E_{C}^{2}}}=2$ for $n=2$, and $\overline{\overline{E_{C}^{3}}}=3$ for $n=3$, it is trivial to induce that $\overline{\overline{E_{C}^{n}}}=n$, where $n \in \mathbf{N}$ and it is the number of mesh dimensions.

\subsubsection{Edge classes $E_{C k}^{n}$}

The cardinality $\overline{\overline{E_{C k}^{n}}}$ for $k=1,2, \cdots, n$ cannot be that trivially induced as $\overline{\overline{E_{C}^{n}}}$. Surprisingly it is however noticed that $\overline{\overline{E_{C k}^{n}}}$ for $n$-mesh is identical to the elements of $n$-cube (see Table 1) divided by 2 , when omitting the last element:

$E_{m, n} / 2=2^{n-m-1}\left(\begin{array}{c}n \\ m\end{array}\right), \quad m=0,1, \cdots, n-1$.

It can be further transformed into more convenient form:

$E_{m, n} / 2=2^{n-m-1}\left(\begin{array}{c}n \\ m\end{array}\right)=2^{k-1}\left(\begin{array}{c}n \\ n-k\end{array}\right)=2^{k-1}\left(\begin{array}{l}n \\ k\end{array}\right)$,

$\overline{\overline{E_{C k}^{n}}}=2^{k-1}\left(\begin{array}{l}n \\ k\end{array}\right), \quad k=1,2, \cdots, n$.

It can be noticed that the form of the formula is not accidental. $\overline{\overline{E_{C k}^{n}}}$ is equal to the multiplication of permutation and combination components, which are respectively $2^{k-1}$ and $\left(\begin{array}{l}n \\ k\end{array}\right)$. The latter one is equal to the number of all $k$-combinations of $n$-dimensions, whereas the former one is equal to the number of all direction permutations of the $(k-1)$-combinations. For each permutation, one dimension is excluded since the graph is undirected. For example for $E_{C}^{3}$ :

\subsubsection{Total number of edge subclasses $\overline{\overline{U E_{C}^{n}}}$}

The formula for total number of edge subclasses can be derived from binomial theorem:

$$
\begin{aligned}
\overline{\overline{\bigcup E_{C}^{n}}} & =\sum_{k=1}^{n} \overline{\overline{E_{C k}}}=\sum_{k=1}^{n} 2^{k-1}\left(\begin{array}{l}
n \\
k
\end{array}\right) \\
& =2^{-1}\left[\sum_{k=0}^{n} 2^{k}\left(\begin{array}{l}
n \\
k
\end{array}\right)-1\right] .
\end{aligned}
$$

$$
\begin{aligned}
(x+y)^{n} & =\sum_{k=0}^{n} x^{n-k} y^{k}\left(\begin{array}{l}
n \\
k
\end{array}\right) \\
& \Rightarrow \sum_{k=0}^{n} 2^{k}\left(\begin{array}{l}
n \\
k
\end{array}\right)=2 \overline{\overline{\sqrt{E_{C}^{n}}}}+1=(1+2)^{n} .
\end{aligned}
$$

$\overline{\overline{\bigcup_{E_{C}^{n}}^{n}}}=\left(3^{n}-1\right) / 2$.

\subsection{Generalized formulas}

In the Sect. 3.2.3 one has derived formulas determining sets of graph edges for graph representation of $n$-mesh (see Table 2):

$\left\{\begin{array}{l}\overline{\overline{E_{C}^{n}}}=n, \\ \overline{\overline{E_{C k}^{n}}}=2^{k-1}\left(\begin{array}{l}n \\ k\end{array}\right), \quad k=1,2, \cdots, n, \\ \overline{\overline{U E_{C}^{n}}}=\left(3^{n}-1\right) / 2 .\end{array}\right.$

\subsection{Graph representation of folded $n$-mesh}

Graph representation can be also created for folded $n$-mesh. Then the peripheral vertices are connected by additional edges, as shown in Fig. 4.

\section{Algorithm}

The algorithm that constructs graph representation for $n$ mesh is shown in Fig. 5. It uses $\left\{M_{n}, d_{f}, E_{u}\right\}$ as input and returns $\{D\}$ as output, where:

$\boldsymbol{M}_{n}$ is an matrix $d_{1} \times \cdots \times d_{n}$ representing $n$-mesh, where $n \geq 2$ and $d_{k} \geq 3$ for $k=1,2, \cdots, n$.

$$
\left\{\begin{array}{lcc}
\overline{\overline{E_{C 1}^{3}}}=2^{0}\left(\begin{array}{l}
3 \\
1
\end{array}\right)=1 * 3 \Rightarrow & E_{C 1}^{3}=\left\{E_{\hat{x}}\right\} \cup\left\{E_{\hat{y}}\right\} \cup\left\{E_{\hat{z}}\right\}, \\
\overline{\overline{E_{C 2}^{3}}}=2^{1}\left(\begin{array}{l}
3 \\
2
\end{array}\right)=2 * 3 \Rightarrow & \Rightarrow & E_{C 2}^{3}=\left\{E_{\hat{x}+\hat{y}}, E_{\hat{x}-\hat{y}}\right\} \cup\left\{E_{\hat{x}+\hat{z}}, E_{\hat{x}-\hat{z}}\right\} \cup\left\{E_{\hat{y}+\hat{z}}, E_{\hat{y}-\hat{z}}\right\}, \\
\overline{\overline{E_{C 3}^{3}}}=2^{2}\left(\begin{array}{l}
3 \\
3
\end{array}\right)=4 * 1 & \Rightarrow & E_{C 3}^{3}=\left\{E_{\hat{x}+\hat{y}+\hat{z}}, E_{\hat{x}+\hat{y}-\hat{z}}, E_{\hat{x}-\hat{y}+\hat{z}}, E_{\hat{x}-\hat{y}-\hat{z}\} .}\right\}
\end{array}\right.
$$


Table 2 Sets of graph edges determined for $n$-mesh, where $n=1,2, \cdots, 6$

\begin{tabular}{|c|c|c|c|c|c|c|c|c|c|}
\hline$\overline{\overline{E_{C k}^{n}}}=2^{k-1}$ & $\left(\begin{array}{l}n \\
k\end{array}\right)$ & & & & & & $E_{C}^{n}=\left\{\overline{\overline{E_{C 1}^{n}}}, \overline{\overline{E_{C 2}^{n}}}, \cdots, \overline{\overline{E_{C n}^{n}}}\right\}$ & $\overline{\overline{E_{C}^{n}}}$ & $\overline{\overline{U E_{C}^{n}}}$ \\
\hline \multirow[t]{2}{*}{$n$} & \multicolumn{6}{|l|}{$k$} & & & \\
\hline & 1 & 2 & 3 & 4 & 5 & 6 & & & \\
\hline 1 & 1 & & & & & & $\{1\}$ & 1 & 1 \\
\hline 2 & 2 & 2 & & & & & $\{2,2\}$ & 2 & 4 \\
\hline 3 & 3 & 6 & 4 & & & & $\{3,6,4\}$ & 3 & 13 \\
\hline 4 & 4 & 12 & 16 & 8 & & & $\{4,12,16,8\}$ & 4 & 40 \\
\hline 5 & 5 & 20 & 40 & 40 & 16 & & $\{5,20,40,40,16\}$ & 5 & 121 \\
\hline 6 & 6 & 30 & 80 & 120 & 96 & 32 & $\{6,30,80,120,96,32\}$ & 6 & 364 \\
\hline
\end{tabular}

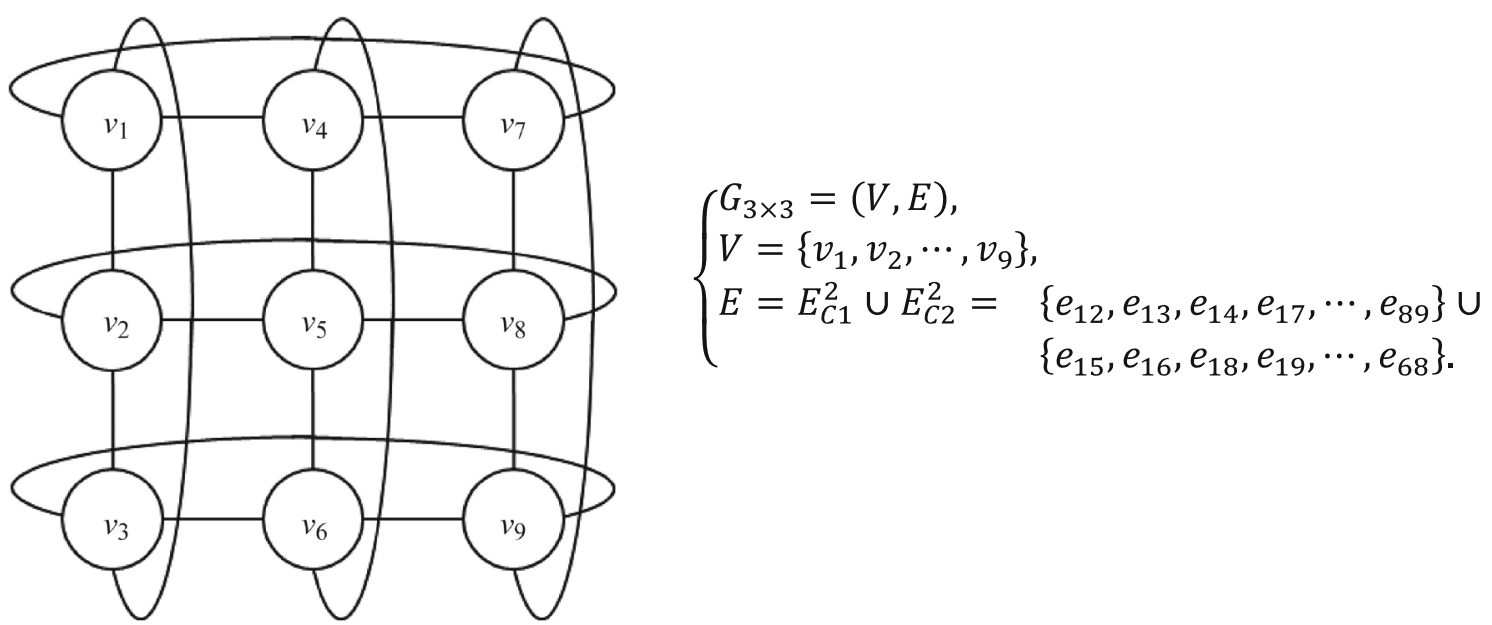

Fig. 4 Graph representation of folded 2-mesh $3 \times 3$ (for clarity, only $E_{C 1}^{2}$ are shown)

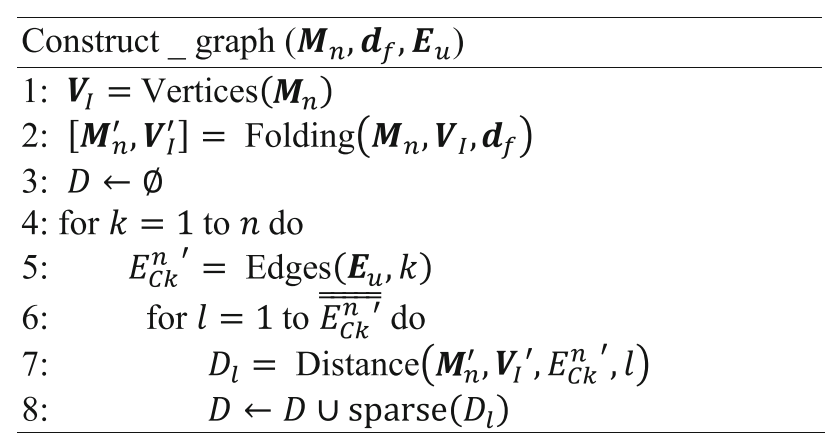

Fig. 5 Pseudo-code of the algorithm constructing graph representation for $n$-mesh

$\boldsymbol{d}_{f}$ is a binary vector $\left[d_{f 1}, d_{f 2}, \cdots, d_{f n}\right]$ determining the dimensions to be folded, where $d_{f k}=1$ if folded, $d_{f k}=0$ if not folded, and $k=1,2, \cdots, n$.

$\boldsymbol{E}_{u}$ is a binary vector $\left[e_{u 1}, e_{u 2}, \cdots, e_{u n}\right]$ determining the edge classes to be used in the graph representation, where $e_{u k}=1$ if used, $e_{u k}=0$ if not used, and $k=1,2, \cdots, n$.
$\boldsymbol{D}$ is a distance matrix $V \times V$, where $V=$ $\left[v_{1}, v_{2}, \cdots, v_{m}\right], m=\prod_{k=1}^{n} d_{k}$, and $d_{k}$ is the number of samples along $k$-dimension of $\boldsymbol{M}_{n}$.

\subsection{Vertices $\left(\boldsymbol{M}_{n}\right)$}

$\boldsymbol{V}_{I}$ is a matrix that has the same size $d_{1} \times d_{2} \times \cdots \times d_{n}$ as $\boldsymbol{M}_{n}$. It contains indexes of graph vertices $\boldsymbol{V}_{I}\left[i_{1}, i_{2}, \cdots, i_{n}\right]$ $=i_{1}+\sum_{k=2}^{n}\left[\left(i_{k}-1\right) \prod_{l=1}^{k-1} d_{l}\right]$.

\subsection{Folding $\left(\boldsymbol{M}_{n}, \boldsymbol{V}_{I}, \boldsymbol{d}_{f}\right)$}

In order to create graph representation for $n$-mesh with folded dimensions, there need to be added edges between vertices representing peripheral samples (see Fig. 4). It is done iteratively by extending $\boldsymbol{M}_{n}$ and $\boldsymbol{V}_{I}$ along dimensions specified in $\boldsymbol{d}_{f}$. For $d_{f k}=1$ and $k=1,2, \cdots, n$ the matrices $\boldsymbol{M}_{n}$ and $\boldsymbol{V}_{\boldsymbol{I}}$ are extended by adding at their end the submatrix $d_{1} \times d_{2} \times \cdots \times d_{k-1} \times 1 \times d_{k+1} \times \cdots \times d_{n}$. It 
results in $\boldsymbol{M}_{n}^{\prime}$ and $\boldsymbol{V}_{I}^{\prime}$ with the size $d_{1} \times d_{2} \times \cdots$ $\times d_{k-1} \times\left(d_{k}+1\right) \times d_{k+1} \times \cdots \times d_{n}$.

For example, if $\boldsymbol{M}_{n}$ has the size $d_{1} \times d_{2}$ and $\boldsymbol{d}_{f}=$ [ 11 1 $]$, the folding is done iteratively by repeating the first column, and then by repeating the first row, hence $\boldsymbol{M}_{n}^{\prime}$ has the size $\left(d_{1}+1\right) \times\left(d_{2}+1\right)$ and the elements:

$$
\begin{aligned}
\boldsymbol{M}_{n}^{\prime} & =\left[\begin{array}{cc}
\boldsymbol{M}_{n} & \boldsymbol{M}_{n}(1, l) \\
\boldsymbol{M}_{n}(k, 1) & \boldsymbol{M}_{n}(1,1)
\end{array}\right], \\
k & =1,2, \cdots, d_{1}, l=1,2, \cdots, d_{2} .
\end{aligned}
$$

\subsection{Edges $\left(\boldsymbol{E}_{u}, k\right)$}

The purpose of Edge is to evaluate edge subclasses for edge classes $E_{C k}^{n}$ (see Sect. 3) specified by $\boldsymbol{E}_{u}$. For example, if $E_{C k}^{n}$ is as in the Sect. 3.2.2 and $\boldsymbol{E}_{u}=\left[\begin{array}{lll}1 & 0 & 1\end{array}\right]$, Edges returns for $k=1$ the list of lists $E_{C 1}^{3 \prime}=[[+\hat{x}],[+\hat{y}],[+\hat{z}]]$, for $k=$ $2: E_{C 2}^{3 \prime}=[]$, and $k=3: E_{C 3}^{3 \prime}=[[+\hat{x},+\hat{y},+\hat{z}],[+\hat{x},+\hat{y},-\hat{z}]$, $[+\hat{x},-\hat{y},+\hat{z}],[+\hat{x},-\hat{y},-\hat{z}]]$.

\subsection{Distances $\left(\boldsymbol{M}_{n}^{\prime}, \boldsymbol{V}_{\mathrm{I}}^{\prime}, E_{C k}^{n \prime}, l\right)$}

The distance between neighboring vertices $v_{i}$ and $v_{j}$ is $D[i, j]=m w$, where $m=\left\|\left(v_{i}, v_{j}\right)\right\|$ is metric and $w$ is weight. For example, if the metric is Euclidean distance and $w=1$, then $D[i, j]=\sqrt{k} \quad$ (see Sect. 2.2) and $k=1,2, \cdots, n$ is the number of dimensions involved in moving between neighboring vertices.

Similarly the distances can be calculated for submatrices of $\boldsymbol{V}_{I}^{\prime}$ and $\boldsymbol{M}_{n}^{\prime}$ through their matrix product $D_{l}=m\left(\boldsymbol{V}_{I}^{\prime}\right) \cdot w\left(\boldsymbol{M}_{n}^{\prime}\right)$, which is further transformed into the sparse form $D_{l}^{\prime}=$ $\operatorname{sparse}\left(V_{I i}^{\prime}, V_{I j}^{\prime}, D_{l}\right)$. For example, if again the metric is Euclidean distance, the weight is exponential of absolute value of gradient. One considers $[+\hat{x},+\hat{y},-\hat{z}]$, which is $(l=2)$ the element of $E_{C 3}^{3 \prime}$ in the Sect. 4.3, then:

$$
\left\{\begin{aligned}
D_{l}^{\prime} & =\operatorname{sparse}\left(V_{I i}^{\prime\left(s_{i}\right)}, V_{I j}^{\prime\left(s_{j}\right)}, D_{l}\right), \\
D_{l} & =\sqrt{\sum_{k=1}^{n}\left(V_{I i}^{\prime}\left(s_{i}\right)-V_{I j}^{\prime}\left(s_{j}\right)\right)^{2}} \cdot \exp \left|M_{n i}^{\prime}\left(s_{i}\right)-M_{n j}^{\prime}\left(s_{j}\right)\right| \\
s_{i} & =\left[1 \cdots d_{1}-1,1 \cdots d_{2}-1,2 \cdots d_{3}\right] \\
s_{j} & =\left[2 \cdots d_{1}, 2 \cdots d_{2}, 1 \cdots d_{3}-1\right] .
\end{aligned}\right.
$$

\section{Application of the algorithm for simple binary 2-mesh $3 \times 3$}

The algorithm is the easiest to understand by considering its main steps for a simple binary 2 -mesh $3 \times 3$ and weight logic function $w=\left(M_{2 i}^{\prime} \cup \boldsymbol{M}_{2 j}^{\prime}\right)^{\prime}$. A binary $n$-mesh is usually used for obstacle avoidance with $\boldsymbol{M}_{2}(i, j)=1$ for an obstacle and $M_{2}(i, j)=0$ for no obstacle. In robotics, such $n$-mesh is known as occupancy grid.
Input

Step 1

Step 2

Step 5

Step 7

$$
\boldsymbol{M}_{2}=\left[\begin{array}{lll}
1 & 1 & 0 \\
0 & 1 & 0 \\
0 & 0 & 1
\end{array}\right], \quad \boldsymbol{d}_{f}=\left[\begin{array}{ll}
1 & 1
\end{array}\right], \quad \boldsymbol{E}_{u}=\left[\begin{array}{ll}
1 & 1
\end{array}\right]
$$

$$
V_{I}=\operatorname{Vertices}\left(\boldsymbol{M}_{2}\right)=\left[\begin{array}{lll}
1 & 4 & 7 \\
2 & 5 & 8 \\
3 & 6 & 9
\end{array}\right]
$$

$$
\left[\boldsymbol{M}_{2}^{\prime}, \boldsymbol{V}_{I}^{\prime}\right]=\text { Folding }\left(\boldsymbol{M}_{2}, \boldsymbol{V}_{I}, \boldsymbol{d}_{f}\right)=\left[\left[\begin{array}{llll}
1 & 4 & 7 & 1 \\
2 & 5 & 8 & 2 \\
3 & 6 & 9 & 3 \\
1 & 4 & 7 & 1
\end{array}\right],\left[\begin{array}{llll}
1 & 1 & 0 & 1 \\
0 & 1 & 0 & 0 \\
0 & 0 & 1 & 0 \\
1 & 1 & 0 & 1
\end{array}\right]\right]
$$

$\{k\}=\{1\}: E_{C k}^{2 \prime}=\operatorname{Edges}\left(\boldsymbol{E}_{u}, k\right)=[[+\hat{x}],[+\hat{y}]]$

$\{k\}=\{2\}: E_{C k}^{2 \prime}=\operatorname{Edges}\left(\boldsymbol{E}_{u}, k\right)=[[+\hat{x},+\hat{y}],[+\hat{x},-\hat{y}]]$

$$
\begin{aligned}
& \{k, l\}=\{1,1\}: D_{l}=\sqrt{1}\left(\left[\begin{array}{lll}
1 & 1 & 0 \\
0 & 1 & 0 \\
0 & 0 & 1
\end{array}\right] \cup\left[\begin{array}{lll}
0 & 1 & 0 \\
0 & 0 & 1 \\
1 & 1 & 0
\end{array}\right]\right)^{\prime}=\left[\begin{array}{lll}
0 & 0 & 1 \\
1 & 0 & 0 \\
0 & 0 & 0
\end{array}\right] \\
& \{k, l\}=\{1,2\}: D_{l}=\sqrt{1}\left(\left[\begin{array}{lll}
1 & 1 & 0 \\
0 & 1 & 0 \\
0 & 0 & 1
\end{array}\right] \cup\left[\begin{array}{lll}
1 & 0 & 1 \\
1 & 0 & 0 \\
0 & 1 & 0
\end{array}\right]\right)=\left[\begin{array}{lll}
0 & 0 & 0 \\
0 & 0 & 1 \\
1 & 0 & 0
\end{array}\right] \\
& \{k, l\}=\{2,1\}: D_{l}=\sqrt{2}\left(\left[\begin{array}{lll}
1 & 1 & 0 \\
0 & 1 & 0 \\
0 & 0 & 1
\end{array}\right] \cup\left[\begin{array}{lll}
1 & 0 & 0 \\
0 & 1 & 0 \\
1 & 0 & 1
\end{array}\right]\right)=\left[\begin{array}{lll}
0 & 0 & 1 \\
1 & 0 & 1 \\
0 & 1 & 0
\end{array}\right] \sqrt{2} \\
& \{k, l\}=\{2,2\}: D_{l}=\sqrt{2}\left(\left[\begin{array}{lll}
1 & 0 & 1 \\
1 & 0 & 0 \\
0 & 1 & 0
\end{array}\right] \cup\left[\begin{array}{lll}
0 & 1 & 0 \\
0 & 0 & 1 \\
1 & 1 & 0
\end{array}\right]\right)^{\prime}=\left[\begin{array}{lll}
0 & 0 & 0 \\
0 & 1 & 0 \\
0 & 0 & 1
\end{array}\right] \sqrt{2}
\end{aligned}
$$




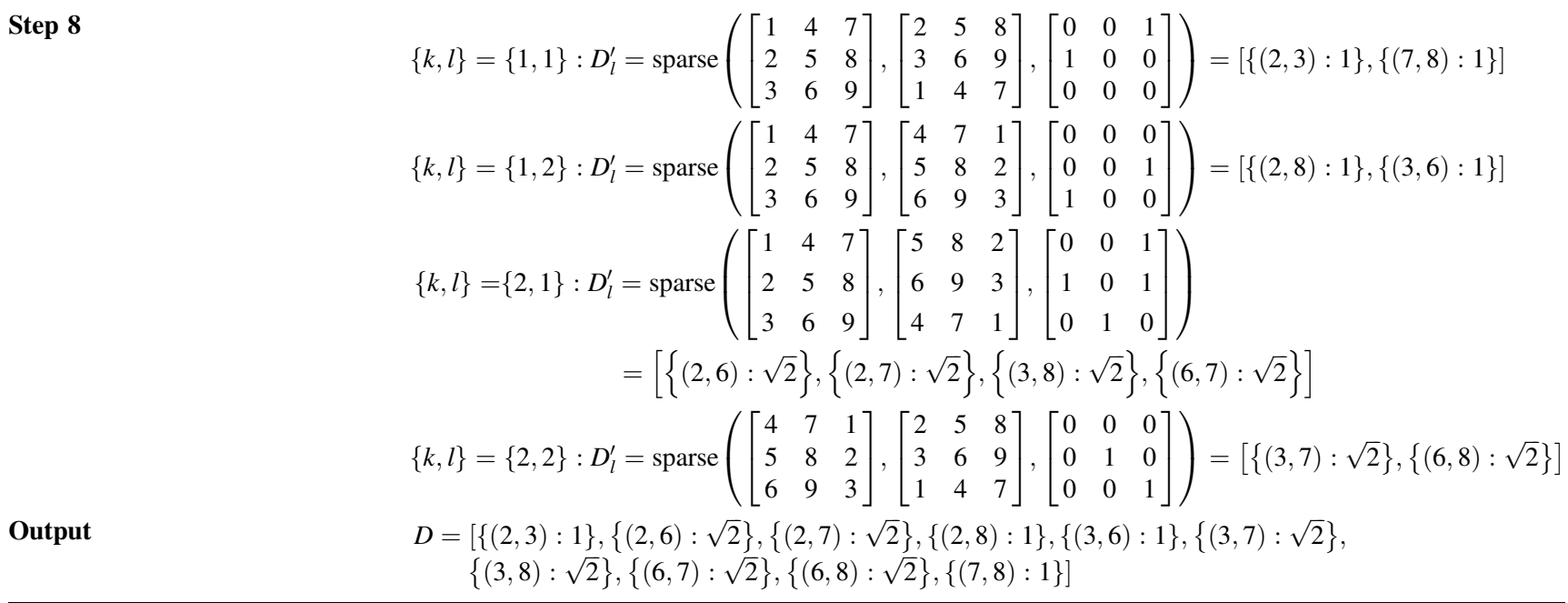

\section{Conclusions}

The paper has investigated how graph representation can be created for mesh that is a discrete approximation of $n$ dimensional continuous space. The mesh that was used is a Sukharev grid [6], which is obtained by uniform deterministic sampling of the space. The investigation has revealed the relationship between mesh dimensionality and the type and quantity of edges that can connect each vertex with its neighbors. There is also proposed simple algorithm to create such graph representations.

Though, there are more efficient sampling methods and enhanced algorithms, the simplicity of used ones allows for better insight of the problem. The analysis can be further extended to cover other sampling methods, including also stochastic and ununiform ones. Similarly there can be computationally proposed much less expensive modifications of the algorithm. Further investigation can also allow plenty of efficient graph search algorithms, which are primarily used for discrete systems, such as ant colony optimization (ACO), to be applied for path and trajectory optimization in continuous systems. While in the investigation it is assumed that the graph representation is created entirely in a single step, one can imagine that the subtasks of sampling space, creating representation, and searching optimal solution are done iteratively in a feedback loop. Then vertices can be adaptively added and removed from the representation, basing for instance on the amount of pheromone left by artificial ants [12].
Acknowledgments I would like to thank Torstein Anderssen Myhre, a PhD student and my colleague at NTNU, who helped me to find and remove inconsistencies in mathematical notation.

\section{References}

1. LaValle SM, Jr Kuffner JJ (2001) Randomized kinodynamic planning. Int J Robot Res 20(5):378-400

2. Applegate DL, Bixby RE, Chvátal V et al (2011) The travelling salesman problem: a computational study. Princeton University Press, Princeton

3. Sánchez G, Latombe JC (2002) Using a PRM planner to compare centralized and decoupled planning for multi-robot system. In: IEEE international conference on robotics and automation, vol 2, pp 2112-2119

4. Carpin S (2006) Randomized motion planning: a tutorial. Int J Robot Autom 21(3):184-195

5. LaValle SM (2006) Planning algorithms. Cambridge University Press, Cambridge

6. Sukharev AG (1971) Optimal strategies of the search for an extremum. USSR Comput Math Math Phys 11(4):910-924

7. Gross JL, Yellen J (2003) Handbook of graph theory. CRC Press, Boca Raton

8. Hogben L (2013) Handbook of linear algebra. 2nd edn. Chapman and Hall/CRC, Boca Raton

9. Goodman JE, O'Rourke J (2004) Handbook of discrete and computational geometry. 2nd edn. Chapman and Hall/CRC, Boca Raton

10. Coxeter HSM (1973) Regular polytopes. 3rd edn. Dover Publications, New York

11. Lee J (2013) Introduction to topological manifolds. 2nd edn. Springer, New York

12. Dorigo M, Maniezzo V, Colorni A (1996) Ant system: optimization by a colony of cooperating agent. IEEE Trans Syst Man Cybern 26(1):29-41 